\title{
Extrusion of unleavened bread dough: experiments and simulations
}

\begin{abstract}
An experimental and numerical study on ram extrusion of bread dough was conducted in order to develop predictive models for the pressures involved, as well as the deformation of the extruded dough. Such studies are needed as high pressures can potentially lead to significant degassing, tearing, and shearing of the dough and hence poor bread quality; the latter limits the use of extrusion processes which would otherwise be a cost-effective forming process. A laboratory extrusion rig was designed, with dies of varying angles and exit radii. Rate dependent behavior was observed from tests conducted at different extrusion speeds, and higher extrusion pressure was reported for dies with smaller exit radius or larger die angle. A simulation of extrusion was performed to predict the extrusionpressure as well as the extrudate swell, as a function of die geometry and extrusion rate. A continuum approach was taken in the constitutive model of dough which is a starch filled system in a protein matrix. A nonlinear viscoelastic model combined for the first time with the Mullins model for filled rubbers is found to capture the continuum behavior well. A Coulomb friction law combined with a maximum shear stress limit was used to describe the contact definition between the extrusion barrel and the dough. Higher die angles and higher extrusion speeds require higher shear stress limit values for the model and the experimentsto agree. A possible reason for this is that the shear stress limit depends on maximum strain as well as strain rate imposed during the extrusion process. Static zones were observed both experimentally and numerically. The onset of the static zones was predicted well but quantifying the geometry of the latter needs further studies.
\end{abstract}

Keyword: Bread dough; Adaptive meshing; Extrusion pressure; Critical shear stress limit; Extrudate swell; Rheology 